ARTICLE

Received 16 Dec 2015 | Accepted 19 Apr 2016 | Published 2 Jun 2016

DOI: $10.1038 /$ ncomms 11698 OPEN

\title{
Fast, greener and scalable direct coupling of organolithium compounds with no additional solvents
}

Erik B. Pinxterhuis ${ }^{1}$, Massimo Giannerini ${ }^{1}$, Valentín Hornillos ${ }^{1} \&$ Ben L. Feringa ${ }^{1}$

Although the use of catalytic rather than stoichiometric amounts of metal mediator in cross-coupling reactions between organic halides and organometallic counterparts improves significantly the atom economy and waste production, the use of solvents and stoichiometric generation of main-group byproducts ( $B, S n$ and $\mathrm{Zn}$ ) hamper the 'greenness' and industrial efficiency of these processes. Here we present a highly selective and green Pd-catalysed cross-coupling between organic halides and organolithium reagents proceeding without additional solvents and with short reaction times $(10 \mathrm{~min})$. This method bypasses a number of challenges previously encountered in $\mathrm{Pd}$-catalysed cross-coupling with organolithium compounds such as strict exclusion of moisture, dilution and slow addition. Operational ease of this protocol combines the use of industrially viable catalysts loadings (down to $0.1 \mathrm{~mol} \%$ ), scalability of the process (tested up to $120 \mathrm{mmol}$ ) and exceptionally favourable environmental impact (E factors in several cases as low as 1).

\footnotetext{
${ }^{1}$ Faculty of Mathematics and Natural Sciences, Stratingh Institute for Chemistry, University of Groningen, Nijenborgh 4, 9747AG Groningen, The Netherlands. Correspondence and requests for materials should be addressed to V.H. (email: v.hornillos.gomez.recuero@rug.nl) or to B.L.F. (email: b.l.feringa@rug.nl).
} 
T he development of greener, more efficient and simple reaction methodologies sets a priority for the synthetic chemistry community both in industry and in academia as well ${ }^{1}$. Solvents are mainly responsible for the environmental impact of synthetic procedures, being, in general, the largest contributors to the magnitude of the $\mathrm{E}$ factor $(\mathrm{E}=$ organic waste $(\mathrm{kg}) /$ product $(\mathrm{kg}))$, a value introduced by Sheldon et al. ${ }^{2}$ to measure the 'greenness' of a chemical process ${ }^{3}$. Thus, reduction or elimination of solvents from organic reactions is of major concern in chemical process development ${ }^{4-7}$. Higher energy needs, toxicity, safety hazards and massive waste treatment are direct implications of the use of large volumes of solvents that negatively affect both costs and environment. Inspired by the 12 principles of Green Chemistry ${ }^{8}$, the development of sustainable products is committed to reduce or, possibly, prevent the use of traditional solvents that still, as today, represent the major share of chemical waste production (up to $80 \%$ ).

An ideal solution to the above-mentioned issues is to completely exclude the solvent from the reaction medium. These so-called solvent-free conditions often lead to additional improvements in other critical parameters as well, such as the catalytic loading (generally lower), the speed of the reaction (generally higher) and the volume/output ratio ${ }^{9}$.

A particular challenging class of transformations in this respect are the widely used transition metal-catalysed reactions. Despite the central role played by Pd-catalysed cross-coupling reactions of organometallic compounds with organo-(pseudo)halides, both in industrial ${ }^{10,11}$ and in academic laboratories ${ }^{12-15}$, the corresponding solvent-free variants have been scarcely reported. Although boron compounds have been engaged in solvent-free cross-coupling reactions, thus far the use of microwaves ${ }^{16,17}$, ball mill ${ }^{18,19}$ and/or high temperatures are required (Fig. 1a).

Our group has recently described methods for the palladium-catalysed direct cross-coupling of highly reactive organolithium reagents ${ }^{20,21}$ (among the most versatile and widely used reagents in organic synthesis) with organic halides under mild conditions ${ }^{22-29}$. The extreme reactivity of organometallic reagents such as organolithium compounds commonly dictates highly delicate conditions such as low temperatures, dilution, slow addition and so on, to achieve high conversion and selectivity in their chemical transformations. In the case of Pd-catalysed cross-coupling reactions directly applying organolithium compounds, the use of toluene as a solvent and slow addition of a previously diluted solution of organolithium reagent are key factors to obtain high selectivity and good yields, while avoiding the notorious lithium-halogen exchange and homocoupling side reactions (Fig. 1b) ${ }^{30-32}$.

Despite the formidable challenge presented by the quest to control selectivity when mixing organolithium reagents with neat organohalides, owing to the possibility for numerous competing reactions, we show here the development of the first Pd-catalysed solvent-free cross-coupling of highly polar organometallic compounds that, through a concise and simple procedure, affords the desired coupled product with excellent selectivities within 10 min and in many cases with E factors as low as 1 (Fig. 1c).

\section{Results}

Preliminary observations. Inspired by the report of García-Álvarez and colleagues ${ }^{33}$ on the use of deep eutectic solvents (DES, mostly obtained by mixing a quaternary ammonium salt as choline chloride with a hydrogen-bond donor such as glycerol or water) for the 1,2-addition of Grignard and organolithium reagents to ketones, we set out to explore the Pd-catalysed cross-coupling reaction of organolithium compounds and organic halides employing these green solvents. Despite the high reactivity of organolithium compounds towards protic solvents, we were delighted to find that the reaction between an excess of PhLi (2-10 eq) and 1-bromonaphthalene using $10 \mathrm{~mol} \%$ of Pd catalyst in a type III DES proceeded with good selectivity, although in low yield (28-53\% conversion, see Supplementary Table 1). We hypothesized that probably small droplets of substrate containing high concentration of catalyst were formed, and that a Established methods

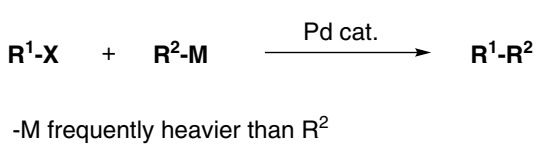

Stille $(\mathbf{M}=\mathbf{S n})$

Negishi $(\mathbf{M}=\mathbf{Z n})$

Suzuki-Miyaura $(\mathbf{M}=\mathbf{B})$

Hiyama-Denmark $(\mathbf{M}=\mathbf{S i})$

Kumada $(\mathbf{M}=\mathbf{M g})$

-Solvent or solvent-free under microwave, ball mill and/or high temp $(\mathbf{M}=\mathbf{B})$

b Our previous work

\begin{tabular}{c|c|c}
$\mathbf{R}^{1}-\mathbf{X}$ & $\begin{array}{c}\mathbf{R}^{2}-\mathbf{L i} \\
\text { - Added over } 1-4 \mathrm{~h} \\
\text { - Has to be diluted }\end{array}$ & Toluene
\end{tabular} $\mathbf{R}^{1}-\mathbf{R}^{2}$

C This work

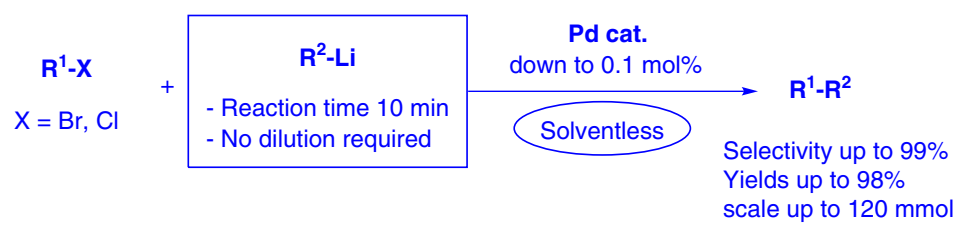

Figure 1 | State-of-the-art overview. (a) Established methods for Pd-catalysed cross-coupling reactions. (b) Catalytic cross-coupling with organolithium compounds. (c) A fast, highly scalable and solvent-free direct cross-coupling of organolithium compounds. 
the reaction was taking place directly in the organic phase rather than in the DES phase. However, owing to quenching of the organolithium reagent by the solvent, the conversions obtained were low. We questioned whether the innate reactivity of organolithium compounds could be turned into an inherent advantage offering the possibility to develop a solvent-free Pd-catalysed cross-coupling protocol, which proceeds within minutes, without the support of any additional device (microwave, ball mills and so on), with low catalytic loading and at ambient temperature without the use of strictly inert conditions.

Reaction conditions optimization. We set out to investigate the reaction between 4-methoxybromobenzene 1a, a reluctant aryl bromide in coupling reactions ${ }^{34}$, and commercially available phenyllithium, as successful conditions for the coupling of these two substrates would most probably apply to a wide variety of other coupling partners as well (Table 1). All the reactions were carried out by adding the organolithium compound (without prior dilution) to a neat mixture of catalyst and organic halide over $10 \mathrm{~min}$ at room temperature (RT). Moreover, we employed a 1 -mmol scale to illustrate the synthetic utility of the method. Reactions using the in situ-prepared palladium complex $\mathrm{Pd} /$ XPhos (generated by mixing $\mathrm{Pd}_{2}(\mathrm{dba})_{3}$ with XPhos) ${ }^{35}$, previously reported to be effective for other Pd-catalysed cross-coupling reactions with aryllithium reagents ${ }^{25,26}$, afforded the crosscoupling product $\mathbf{2 a}(R=\mathrm{Ph})$ within $10 \mathrm{~min}$, although in the presence of significant amounts of the undesired homocoupling product (Table 1, entry 1). By employing Pd-PEPPSI-IPent catalyst ${ }^{36}$, the selectivity was raised to $90 \%$ at the expense of the homocoupling product (Table 1, entry 2). Nonetheless, we were delighted to find that the commercially available air- and temperature-stable Pd-PEPPSI-IPr catalyst, which is seven times cheaper than Pd-PEPPSI-IPent ${ }^{37}$, afforded full conversion and nearly perfect selectivity $(>95 \%$,) towards the coupled product 2a $(R=\mathrm{Ph})$ at $\mathrm{RT}$ in $<10 \mathrm{~min}$, avoiding the formation of dehalogenation or homocoupling side products 3 and $\mathbf{4}$ (Table 1, entry 3). Importantly, the high selectivity was maintained while lowering the catalyst loading to $1.5 \mathrm{~mol} \%$ (Table 1 , entry 4 , $84 \%$ isolated yield). With an efficient catalyst for $C s p^{2}-C s p^{2}$ cross-coupling in hands, we then turn our attention to the challenging $C s p^{3}-C s p^{2}$ solvent-free cross-coupling with alkyllithium compounds. The direct use of commercially available $n-\mathrm{BuLi}$, one of the most reactive organometallic reagents, in combination with Pd-PEPPSI-IPr led to the desired product $2 \mathbf{v}(R=n-\mathrm{Bu})$, although with slightly diminished selectivity (Table 1, entry 5). Further screening of catalysts showed that the use of commercially available $\operatorname{Pd}\left[\mathrm{P}(t \mathrm{Bu})_{3}\right]_{2}$ catalyst $^{38}$ restored the selectivity $(>95 \%)$ towards the coupled product $2 \mathbf{v}(R=n-\mathrm{Bu})$ with excellent $(82 \%)$ isolated yield (Table 1, entry 6). Importantly, when this reaction was performed using an extremely low catalyst loading $(0.1 \mathrm{~mol} \%)$, product $2 \mathbf{v}(R=n-\mathrm{Bu})$ was still obtained in high conversion and selectivity (Entry 7 ).

Scope and applicability. To our delight, the optimized conditions proved to be general and could be applied successfully to the solvent-free cross-coupling of a variety of aryllithium (2a-2u)

Table 1 | Screening of different ligands.

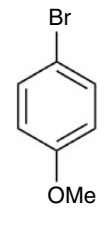

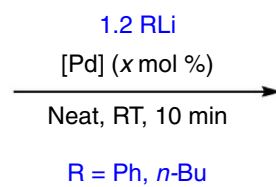

1a
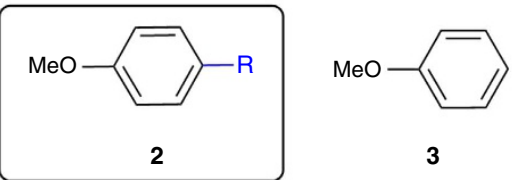

3

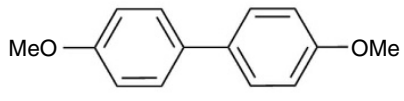

4<smiles>CCCc1cc(Br)c(-c2ccccc2OCC)c(Br)c1</smiles>

XPhos

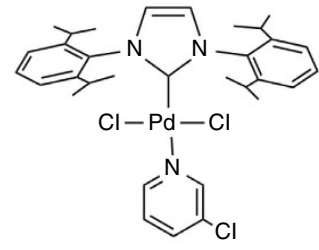

Pd-PEPPSI-IPr

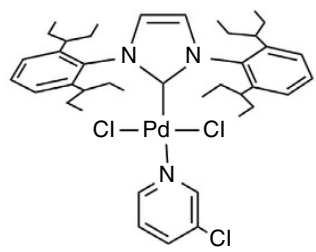

Pd-PEPPSI-IPent

\begin{tabular}{|c|c|c|c|}
\hline Entry $^{\star}$ & $\boldsymbol{R}$ & [Pd] & [Pd] $(x$ \\
\hline 1 & $\mathrm{Ph}$ & $\mathrm{Pd}_{2}(\mathrm{dba})_{3} /$ XPhos & 3 \\
\hline 2 & $\mathrm{Ph}$ & Pd-PEPPSI-IPent & \\
\hline 3 & $\mathrm{Ph}$ & Pd-PEPPSI-IPr & \\
\hline 4 & $\mathrm{Ph}$ & Pd-PEPPSI-IPr & 1. \\
\hline 5 & $n-B u$ & Pd-PEPPSI-IPr & | \\
\hline 6 & $n-B u$ & $\mathrm{Pd}\left[\mathrm{P}(t-\mathrm{Bu})_{3}\right]_{2}$ & 1 \\
\hline 7 & $n-B u$ & $\mathrm{Pd}\left[\mathrm{P}(t-\mathrm{Bu})_{3}\right]_{2}$ & 0 \\
\hline $\begin{array}{l}\text { dba, diben } \\
{ }^{\star} \text { Conditior } \\
+\mathbf{2 : 3 : 4} \text { Ra } \\
\$ 84 \% \text { Yie } \\
\$ 82 \% \text { Yiel } \\
\| 91 \% \text { Con }\end{array}$ & reagen & a mixture of $\mathbf{1}(1 \mathrm{mmol}$ & over 10 min. \\
\hline
\end{tabular}


Table 2 | Pd-Catalysed cross-coupling of organolithium reagents with aryl halides under solventless conditions ${ }^{\star}-I I$.

RLi

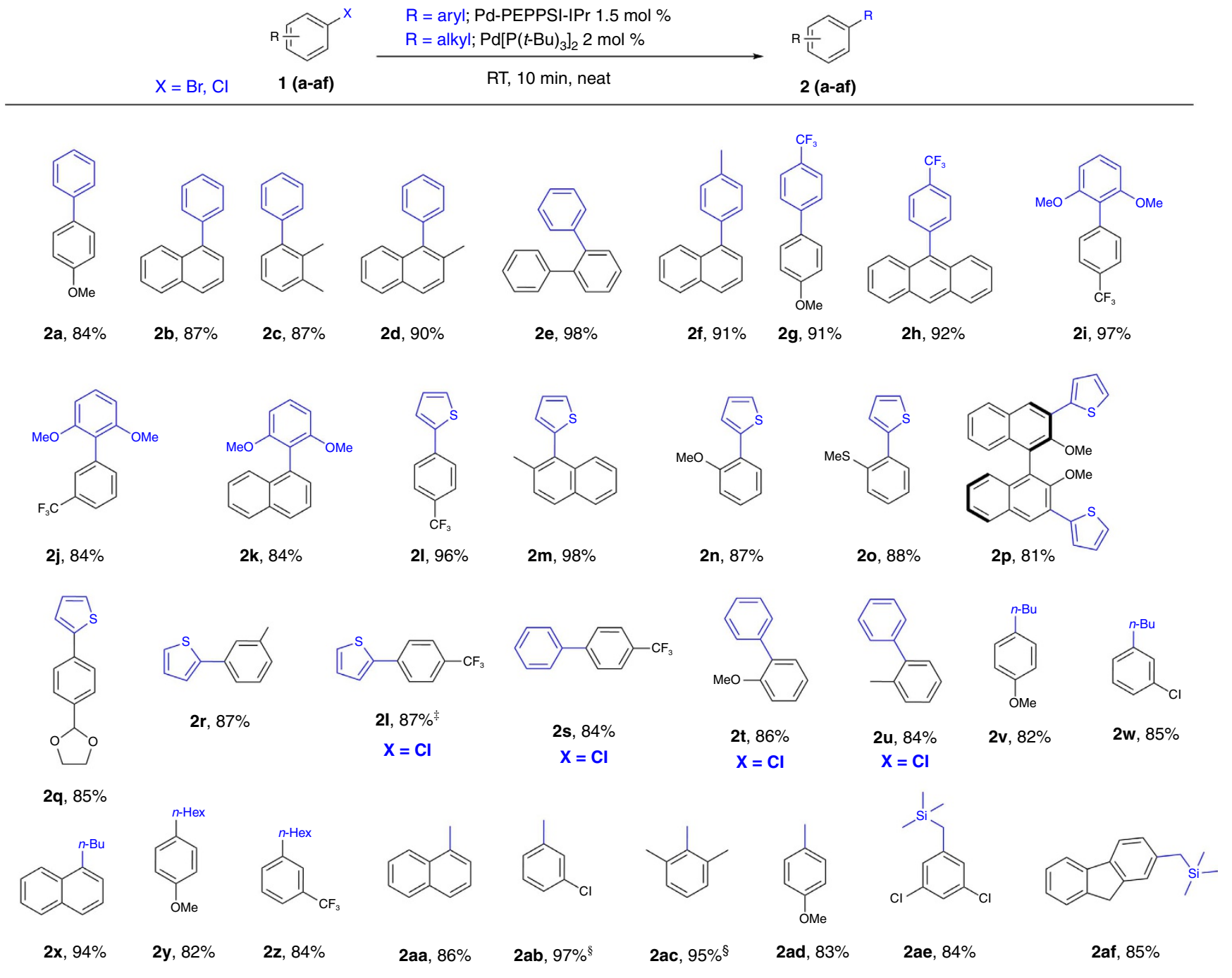

"Conditions: RLi $(1.2 \mathrm{mmol})$ was added to a mixture of organic bromide $(1 \mathrm{mmol})$ and catalyst over $10 \mathrm{~min} . \mathrm{X}, \mathrm{Br}$ unless otherwise noted $(\mathbf{1 1}, \mathbf{1 s}, \mathbf{1 t}, \mathbf{1 u} ; X=\mathrm{Cl})$. Selectivity $2: 3,4>95 \%$. Compound $\mathbf{2}$ was extracted with $\mathrm{Et}_{2} \mathrm{O}$ or $\mathrm{AcOEt}$, after quenching the reaction mixture with minimum amount of sat. aq. $\mathrm{NH}_{4} \mathrm{Cl}$.

$\dagger$ All yields given are isolated yields.

$\$ 3 \mathrm{Mol} \%$ of catalyst was employed for the reaction with 2-thienyllithium.

$\$ G C$ yield: product was not isolated due to volatility issues.

||For limitations of the method, see Supplementary Fig. 1.

and the even more reactive alkyllithium reagents (2v-2af), in all cases affording the products with high selectivity within minutes (Table 2). The remarkably fast cross-coupling methodology gave excellent results in combination with non-commercially available aryllithium reagents obtained through common preparative procedure, such as lithium/halogen exchange (2f-2h) and ortho-directed lithiation. Illustrative is the case of the highly hindered bis-ortho-substituted 2,6-dimethoxy-phenyllithium, used in the synthesis of compounds $2 \mathbf{i}-\mathbf{2} \mathbf{k}$, which was prepared by direct metalation of 1,3-dimethoxybenzene. In all cases, the organolithium reagents were prepared using the minimal amount of ethereal solvent to maintain them soluble (see Supplementary Methods). Despite the higher reactivity and basicity of alkyllithium reagents when compared with (hetero)aryllithium compounds, we were delighted to find high selectivities and yields also for a variety of Csp $p^{3}-\mathrm{C} s p^{2}$ cross-coupling products. This includes the use of different alkyllithium compounds as $n-\mathrm{BuLi}$,
$n$-HexLi and the smallest MeLi with electron-rich and electron-poor arylbromides (Table 2, 2v-2ad). The bifunctional $\mathrm{C}\left(s p^{3}\right)$-(trimethylsilyl)methyllithium reagent ${ }^{28}$ also couples with excellent selectivity, providing synthetically versatile benzylsilanes 2ae and 2af. The lack of dehalogenated side products from these $\mathrm{C} s p^{3}-\mathrm{Cs} p^{2}$ cross-coupling reactions demonstrates that no competing $\beta$-hydride elimination/olefin dissociation ${ }^{24}$ occurs (which is another competing pathway besides the formation of dehalogenated and isomerized products). A limitation so far for this protocol employing the $\operatorname{Pd}\left[\mathrm{P}(t-\mathrm{Bu})_{3}\right]_{2}$-based catalytic system is that secondary alkyllithium reagents such as $i$-PrLi and $s$-BuLi led to the formation of dehalogenation products.

Despite the conditions of highly concentrated reaction partners, various observations highlight how the reaction proceeds exclusively under catalyst control. Thus, the reaction of 1-bromonaphthalene $\mathbf{1 b}$ resulted, with both aryl- and alkyllithium, in the corresponding coupled products $(\mathbf{2 b}, \mathbf{2 f}$, 
$2 \mathbf{k}, 2 \mathrm{x}$ and $2 \mathrm{aa}$ ) without the formation of regioisomers, indicating that benzyne intermediates via 1,2-elimination are not formed. Apart from liquid substrates, even solid bromofluorene 1af was successfully employed, despite the acidity of the benzylic protons $\left(\mathrm{p} K_{\mathrm{a}}=22\right)$. The reaction of $n$-BuLi and $\mathrm{MeLi}$ with p-chloro-bromobenzene occurs selectively with no detectable chloride displacement (Table 2, compounds $2 \mathbf{w}$ and $2 \mathbf{a b}$ ).

Sterically hindered bromides 1c-1e, known for being more reluctant substrates in the synthesis of biaryls ${ }^{23}$, were also successfully coupled at RT in $10 \mathrm{~min}$, indicating that the transmetallation step takes place rapidly, under these conditions, inducing a fast coupling process.

The dramatic effect of the solvent-free conditions in enhancing the reaction rate was demonstrated in the coupling of commercially available 2-thienyllithium, which, according to our previous observations, required the addition of stoichiometric amounts of tetramethylethylenediamine as the activating agent and elevated temperatures $\left(40^{\circ} \mathrm{C}\right)$ to react ${ }^{22}$. Under solvent-free conditions, 2-thienyllithium reacted smoothly at RT within $10 \mathrm{~min}$, in high selectivity and yield, without the use of any additive (see compounds $2 \mathbf{l}-2 \mathbf{r}$ ). We have recently showed that the cross-coupling of 2-alkoxy-substituted arylbromides with organolithium is plagued by fast bromine-lithium exchange induced by the ortho-directing alkoxy unit. The use of the corresponding aryl chlorides, inherently less prone to halogen/ lithium exchange, is thus mandatory, to afford selectively the product and prevent side products formation ${ }^{27}$. However, to our surprise, under our solvent-free protocol, aryl bromides 1n, 1o and $3,3^{\prime}$-dibromo-BINOL 1p, could all be coupled successfully with 2-thienyllithium in high selectivity (>95\%) and with excellent yield, avoiding the notorious bromine-halogen exchange (Table 2). To emphasize the versatility of the new method, it has to be noted that the only previous reported synthesis of BINOL derivate $2 \mathbf{p}$ required the preparation of the corresponding bis-trifluoroboronate BINOL derivative and further reaction with 2-bromothiophene under microwave conditions $^{39}$. The use of acetal-protected aldehyde $\mathbf{1 q}$ was also tolerated without the cleavage of the protecting group. As in the case of alkyllithium compounds, 1-bromo-3-chlorobenzene 1r reacted with 2-thienyllithium using Pd-PEPPSI-IPr catalyst selectively, leaving the chloride untouched. Nevertheless, the electron-poor aryl chloride $\mathbf{1 l}$ reacted readily with 2-thienyllithium and electron-rich chlorides $\mathbf{1 t}$ and $\mathbf{1 u}$ were also easily coupled under the optimized conditions using more reactive $\mathrm{PhLi}$ at $\mathrm{RT}$ (Table 2 ).

A major issue often associated with solvent-free reactions is the homogeneity of the reaction medium, in particular with solid starting materials. However, the methodology presented here provides high selectivity and yields when solid substrates such as 1h, 1p, 1q, lae and 1af were used in combination with aryllithium and $\mathrm{TMSCH}_{2} \mathrm{Li}$ compounds. Unfortunately, the presence of dehalogenated product was observed for the use of $n$-HexLi and $n$-BuLi in combination with solid substrates (for preliminary results using Grignard reagents in similar conditions, see Supplementary Fig. 2).

Scalability of the protocol. In organic chemistry, problems in the scaling up of batch reactions have been known to arise from various issues including inefficient mixing and lack of heat transfer. To test whether this novel method is suitable to be performed on a larger scale, the cross-coupling between $n$-BuLi and 1-bromonaphthalene $\mathbf{1 b}$ was tested on multigram scale with catalyst loading as low as $0.1 \mathrm{~mol} \%$. We were pleased to find that the scale of the reaction had little effect on the selectivity, although the presence of a small amount of dehalogenated side product was observed (Table 3 ). It is noteworthy that the cross-coupling was found to maintain its effectiveness even at $120 \mathrm{mmol}$ scale employing $0.4 \mathrm{~mol} \%$ of catalyst, providing exceptional $\mathrm{E}$ factors as low as 0.8 (Table 3, entry 3 ). It should be emphasized that typical $\mathrm{E}$ factors in the range of 5-100 are seen in transformations producing fine chemicals and pharmaceuticals ${ }^{2}$. Importantly, after the addition of the organolithium compound, the crude product was quenched, washed with water and dried, giving the desired product in reagent-grade quality within $60 \mathrm{~min}$, including all the operations.

Potential of the methodology in synthetic application. To demonstrate the advantages of the new method, we have compared it with some established cross-coupling methodologies currently used in the production of two typical building blocks for pharmaceuticals and conjugated polymers for light-emitting devices. The first example deals with the preparation of a key intermediate (2ag) for the synthesis of a patented melanin concentrating hormone receptor ligand (Fig. $2 a)^{40}$ involved in the treatment of eating disorders, weight gain, obesity, depression and anxiety. The reaction between 1-bromo-4-chlorobenzene 1 ag and 2-thienyllithium under the optimized reaction conditions provided the cross-coupling product 2 ag in high yield and selectivity within $10 \mathrm{~min}$ at RT (E factor: 5.4). In sharp contrast, the reported procedure (E factor: 41 ) involves the corresponding thienylboronic acid, needs a mixture of $\mathrm{DME} / \mathrm{H}_{2} \mathrm{O}$ heated at reflux for $4 \mathrm{~h}, 2 \mathrm{eq}$ of base and requires the corresponding highly reactive aryl iodide. As noted by Lipschutz et al. ${ }^{3}$, aqueous

Table 3 | Catalyst-loading effect for the cross-coupling of 1-bromonaphthalene with $n$-BuLi.

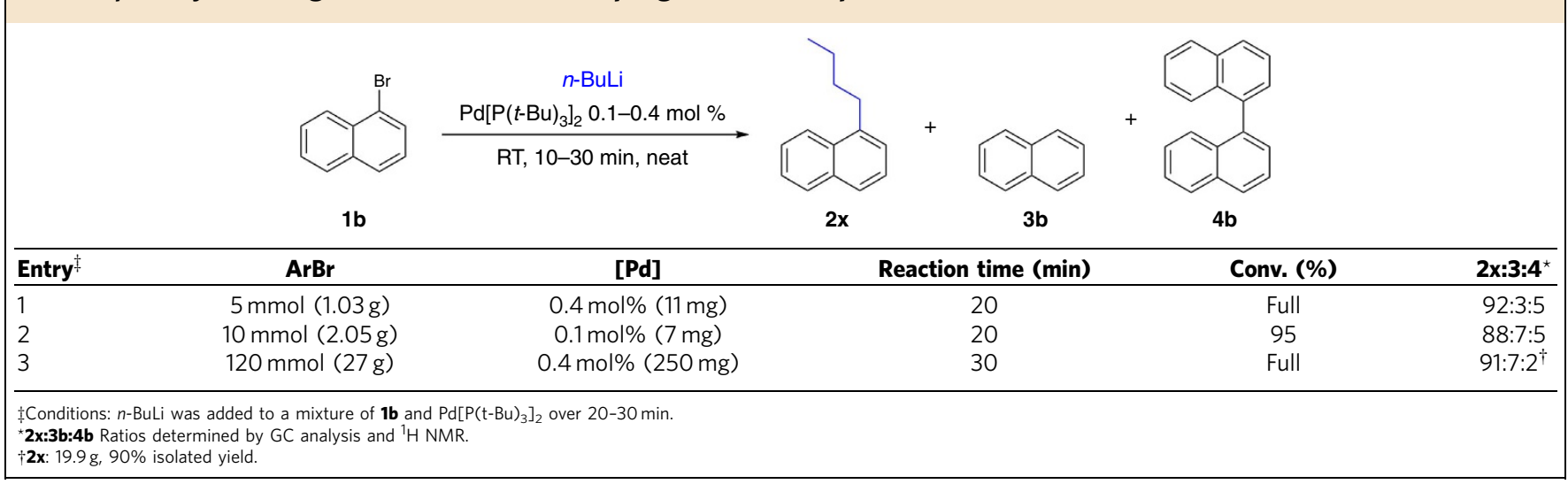


a

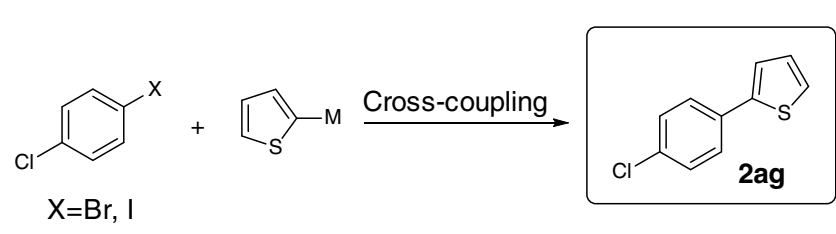

LITERATURE

$\mathrm{M}: \mathrm{B}(\mathrm{OH})_{2}(\mathrm{MW}: 44.8)$
$\mathrm{X}=\mathrm{I}$
$\mathrm{Pd}\left(\mathrm{PPh}_{3}\right)_{4} 5 \mathrm{~mol} \%, \mathrm{Na}_{2} \mathrm{CO}_{3} 2.1$ eq
$\mathrm{DME} / \mathrm{H}_{2} \mathrm{O} 1: 1$, reflux.
Reaction time: $4 \mathrm{~h}$
Yield: $80 \%$
E factor: 41

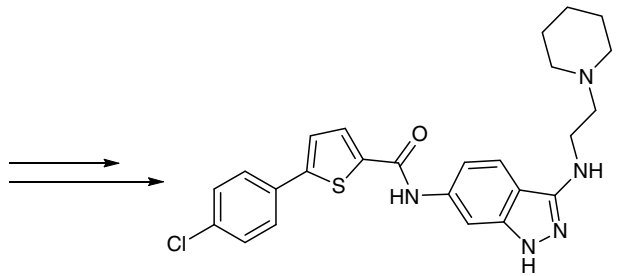

Patented melanin concentrating hormone receptor ligand

\section{THIS WORK}

\author{
M: Li (AW: 6.94) \\ $\mathrm{X}=\mathrm{Br}$ \\ Pd-PEPPSI-IPr 3 mol\% \\ Solvent free, room temperature \\ Reaction time: $10 \mathrm{~min}$ \\ Yield: $97 \%$ \\ E factor: 5.4
}
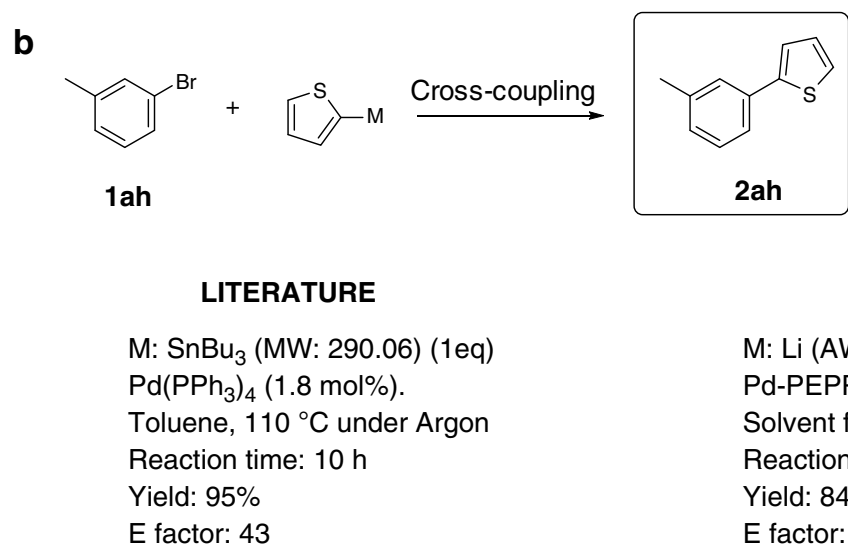

Heteroaromatic-conjugated polymer for optoelectronics

E factor: 43

\title{
THIS WORK
}

\author{
M: Li (AW: 6.94) (1.1 eq) \\ Pd-PEPPSI-IPr 3 mol\% \\ Solvent free, room temperature, in the air \\ Reaction time: $10 \mathrm{~min}$ \\ Yield: $84 \%$ \\ E factor: 8.3
}

Figure 2 | Synthetic applicability. Comparison between established methods and the present cross-coupling protocol with organolithium reagents in the synthesis of key intermediates for a melanin-concentrating hormone receptor ligand (a) and conjugated polymer for optoelectronic devices (b).

workup may also be taken into account in the calculation of the E-factor. In this case, the advantages of using low amounts of solvents become even more evident: although the preparation of 2ag through conventional Suzuki cross-coupling has an E-factor as high as 84 , taking into account the extraction workup, thanks to the reduced amounts of extraction volumes needed, the E-factor obtained for our protocol is 15.4 , marking a dramatic difference of 68.6 units between the two protocols. Notably, LiBr is the major byproduct of the reaction.

The second example illustrates the synthesis of a heteroaromatic monomer employed in the preparation of polymeric materials for optoelectronic devices (Fig. 2b) (ref. 41). Coupling between 2-thienyllithium and 3-methyl-1-bromobenzene $\mathbf{l a h}$ gives access to the desired compound $\mathbf{2 a h}$, at RT, in very good yield within $10 \mathrm{~min}$ and with an $\mathrm{E}$ factor five times lower than that reported in synthetic methodology $\left(110^{\circ} \mathrm{C}, 10 \mathrm{~h}\right.$ in toluene using an organotin compound with a molecular weight four times higher than that of 2-thienyllithium). In the last example, in addition to the remarkable differences in reaction time (10 versus $600 \mathrm{~min})$ and temperature $\left(20^{\circ} \mathrm{C}\right.$ versus $\left.110^{\circ} \mathrm{C}\right)$, the use of solvent-free cross-coupling of 2 -thienyllithium also avoids potentially toxic tin wastes and their often difficult removal, and prevents the use of strictly inert atmosphere required for the coupling of the corresponding tin reagent.

\section{Discussion}

We have discovered that, in sharp contrast to all common reaction protocols using highly reactive organometallics such as organolithium compounds, the Pd-catalysed cross-coupling of arylbromides and alkyl- or aryllithium reagents under neat conditions proceeds exceptionally fast and is selective. Based on this finding we have developed a general solvent-free methodology for the Pd-catalysed direct cross-coupling of organolithium compounds with organic halides, under ambient conditions, providing high yields and excellent selectivities. Fast reaction times (10 $\mathrm{min})$, lower catalyst loading (down to $0.1 \mathrm{~mol} \%$ ), high scalability, operational simplicity (see Methods) and the possibility to avoid the use of a strictly inert atmosphere, of syringe pumps and of additives such as tetramethylethylenediamine are key feature of this methodology. Compared with reported Pd-catalysed cross-coupling, this methodology is particularly attractive, owing to the strongly reduced environmental impact, that is, outstanding volume:time:output ratio, limited amount and low toxicity of the waste and five- to tenfold reduction in E-factor. The use of stable and commercially available catalysts, commercial or readily available and inexpensive organolithium reagents and the applicability to a wide variety of organic bromides are additional factors that contribute to the prospect of these $\mathrm{C}-\mathrm{C}$ bond formations in the art of synthesis. For supporting videos illustrating the ease of operation, see Supplementary Movies 1-2. 


\section{Methods}

The corresponding organolithium reagent $(1.2 \mathrm{eq})$ was added over a mixture of substrate (1.0 eq) and catalyst (1.5-3 mol\%) at RT for $10 \mathrm{~min}$. After the addition was completed, a saturated solution of aqueous $\mathrm{NH}_{4} \mathrm{Cl}$ was added and the mixture was extracted with $\mathrm{AcOEt}$ or $\mathrm{Et}_{2} \mathrm{O}$. The organic phases were combined and dried with anhydrous $\mathrm{Na}_{2} \mathrm{SO}_{4}$. Evaporation of the solvent under reduced pressure afforded the crude product that was then filtered over a silica gel plug to afford the pure product. For NMR spectra of the compounds in this article, see Supplementary Figs 3-73.

Data availability. The authors declare that the data supporting the findings of this study are available within the article and its Supplementary Information files.

\section{References}

1. Dach, R., Song, J. J., Roschangar, F., Samstag, W. \& Senanayake, C. H. The eight criteria defining a good chemical manufacturing process. Org. Process Res. Dev. 16, 1697-1706 (2012).

2. Sheldon, R. A., Arends, I. W. C. E. \& Hanefeld, U. Green Chemistry and Catalysis (Wiley-VCH, 2007).

3. Lipshutz, B. H., Isley, N. A., Fennewald, J. C. \& Slack, E. D. On the way towards greener transition-metal-catalyzed processes as quantified by E factors. Angew. Chem. Int. Ed. 52, 10952-10958 (2013).

4. Tanaka, K. \& Toda, F. Solvent-Free Organic Synthesis (Wiley-VCH, 2003).

5. Nelson, W. M. Green Solvents for Chemistry-Perspectives and Practice (Oxford Univ. Press, 2003).

6. Li, C.-J. Organic reactions in aqueous media with a focus on carbon-carbon bond formations: a decade update. Chem. Rev. 105, 3095-3166 (2005).

7. Simon, M. O. \& Li, C.-J. Green chemistry oriented organic synthesis in water. Chem. Soc. Rev. 41, 1415-1427 (2012).

8. Anastas, P. T. \& Warner, J. C. Green Chemistry: Theory and Practice (Oxford Univ. Press, 1998)

9. Singh, M. S. \& Chowdhury, S. Recent developments in solvent-free multicomponent reactions: a perfect synergy for eco-compatible organic synthesis. RSC Adv. 2, 4547-4592 (2012).

10. Magano, J. \& Dunetz, J. R. Large-scale applications of transition metalcatalyzed couplings for the synthesis of pharmaceuticals. Chem. Rev. 111, 2177-2250 (2011).

11. Topics in Organometallic Chemistry (eds Beller, M. \& Blaser, H. -U.) Vol. 42 (Springer, 2012).

12. Metal-Catalyzed Cross-Coupling Reactions (eds de Meijere, A. \& Diederich, F.) (Wiley-VCH, 2004).

13. Transition Metals for Organic Synthesis (eds Beller, M. \& Bolm, C. (Wiley-VCH, 2004)

14. Handbook of Organopalladium Chemistry for Organic Synthesis (ed. Negishi, E.-I.) (Wiley-Interscience, 2002).

15. Wu, X.-F., Anbarasan, P., Neumann, H. \& Beller, M. From noble metal to Nobel Prize: palladium-catalyzed coupling reactions as key methods in organic synthesis. Angew. Chem. Int. Ed. 49, 9047-9050 (2010).

16. Brooker, M. D., Cooper, Jr S. M., Hodges, D. R., Carter, R. R. \& Wyatt, J. K. Studies of microwave-enhanced Suzuki-Miyaura vinylation of electron-rich sterically hindered substrates utilizing potassium vinyltrifluoroborate. Tetrahedron Lett. 51, 6748-6752 (2010).

17. El Akkaoui, A., Berteina-Raboin, S., Mouaddib, A. \& Guillaumet, G. Direct arylation of imidazo[1,2-b]pyridazines: microwave-assisted one-pot suzuki coupling/Pd-catalysed arylation. Eur. J. Org. Chem. 862-871 (2010).

18. Bernhardt, F., Trotzki, R., Szuppa, T., Stolle, A. \& Ondruschka, B. Solvent-free and time-efficient Suzuki-Miyaura reaction in a ball mill: the solid reagent system KF-Al2O3 under inspection. Beilstein J. Org. Chem. 6, 7 (2010).

19. Schneider, F. \& Ondruschka, B. Mechanochemical solid-state Suzuki reactions using an in situ generated base. ChemSusChem 1, 622-625 (2008).

20. The Chemistry of Organolithium Compounds (ed. Rappoport, Z. \& Marek, I.) (Wiley-VCH, 2004).

21. Lithium Compounds in Organic Synthesis (ed. Luisi, R. \& Capriati, V.) (Wiley$\mathrm{VCH}, 2014)$

22. Giannerini, M., Fañanás-Mastral, M. \& Feringa, B. L. Direct catalytic cross-coupling of organolithium compounds. Nat. Chem. 5, 667-672 (2013).

23. Giannerini, M., Hornillos, V., Vila, C., Fañanás-Mastral, M. \& Feringa, B. L. Hindered aryllithium reagents as partners in palladium-catalyzed crosscoupling: synthesis of tri- and tetra-ortho-substituted biaryls under ambient conditions. Angew. Chem. Int. Ed. 52, 13329-13333 (2013).

24. Vila, C., Giannerini, M., Hornillos, V., Fañanás-Mastral, M. \& Feringa, B. L. Palladium-catalysed direct cross-coupling of secondary alkyllithium reagents. Chem. Sci. 5, 1361-1367 (2014).

25. Hornillos, V., Giannerini, M., Vila, C., Fañanás-Mastral, M. \& Feringa, B. L. Direct catalytic cross-coupling of alkenyllithium compounds. Chem. Sci. $\mathbf{6}$, 1394-1398 (2015).
26. Hornillos, V., Giannerini, M., Vila, C., Fañanás-Mastral, M. \& Feringa, B. L. Catalytic direct cross-coupling of organolithium compounds with aryl chlorides. Org. Lett. 15, 5114-5511 (2013).

27. Castelló, L. M. et al. Pd-catalyzed cross-coupling of aryllithium reagents with 2-alkoxy-substituted aryl chlorides: mild and efficient synthesis of 3,3'-diaryl BINOLs. Org. Lett. 17, 62-65 (2015).

28. Heijnen, D., Hornillos, V., Corbet, B. P., Giannerini, M. \& Feringa, B. L. Palladium-catalyzed C(sp3)-C(sp2) cross-coupling of (trimethylsilyl) methyllithium with (hetero)aryl halides. Org. Lett. 17, 2262-2265 (2015).

29. Vila, C., Hornillos, V., Giannerini, M., Fañanás-Mastral, M. \& Feringa, B. L. Palladium-catalysed direct cross-coupling of organolithium reagents with aryl and vinyl triflates. Chem. Eur. J. 20, 13078-13083 (2014).

30. Pace, V. \& Luisi, R. Expanding the synthetic portfolio of organolithiums: direct use in catalytic cross-coupling reactions. ChemCatChem 6, 1516-1519 (2014).

31. Capriati, V., Perna, F. M. \& Salomone, A. 'The great beauty' of organolithium chemistry: a land still worth exploring. Dalton Trans. 43, 14204-14210 (2014).

32. Firth, J. D. \& O'Brien, P. Cross-Coupling knows no limits: assessing the synthetic potential of the palladium-catalysed cross-coupling of organolithiums. ChemCatChem 7, 395-397 (2015).

33. Vidal, C., García-Álvarez, J., Hernán-Gómez, A., Kennedy, A. R. \& Hevia, E. Introducing deep eutectic solvents to polar organometallic chemistry: chemoselective addition of organolithium and grignard reagents to ketones in air. Angew. Chem. Int. Ed. 53, 5969-5973 (2014).

34. Denmark, S. E., Smith, R. C., Tau, W. T. \& Muhuhi, J. M. Cross-coupling reactions of aromatic and heteroaromatic silanolates with aromatic and heteroaromatic halides. J. Am. Chem. Soc. 131, 3104-3118 (2009).

35. Martin, R. \& Buchwald, S. Palladium-catalyzed Suzuki - Miyaura crosscoupling reactions employing dialkylbiaryl phosphine ligands. Acc. Chem. Res. 41, 1461-1473 (2008).

36. Valente, C. et al. The development of bulky palladium NHC complexes for the most-challenging cross-coupling reactions. Angew. Chem. Int. Ed. 51, 3314-3332 (2012).

37. Source: www.sigmaaldrich.com. (The Netherlands) (October 2015).

38. Fu, G. C. The development of versatile methods for palladium-catalyzed coupling reactions of aryl electrophiles through the use of $\mathrm{P}(\mathrm{t}-\mathrm{Bu}) 3$ and $\mathrm{PCy} 3$ as ligands. Acc. Chem. Res. 41, 1555-1564 (2008)

39. Recsei, C. \& McErlean, S. P. Synthesis of modified binol-phosphoramidites. Tetrahedron 68, 464-480 (2012).

40. Ronsheim, M. \& Araldi, G.-L. Indazole derivatives useful as melanin concentrating receptor ligands. PCT patent application WO 2008061109 (A2) (2008).

41. Kawamoto, K. \& Tominaga, T. Light emitting element. JP2008306170 (A) (2008)

\section{Acknowledgements}

This work was supported financially by the European Research Council (Advanced Investigator Grant Number 227897 to B.L.F.). The Netherlands Organization for Scientific Research (NWO-CW); funding from the Ministry of Education, Culture and Science (Gravitation programme 024.001.035), The Royal Netherlands Academy of Arts and Sciences (KNAW) and NRSC-Catalysis are gratefully acknowledged.

\section{Author contributions}

E.B.P., M.G. and V.H. carried out the experimental work. V.H., M.G. and B.L.F. wrote the manuscript. B.L.F. and V.H. guided the research.

\section{Additional information}

Supplementary Information accompanies this paper at http://www.nature.com/ naturecommunication

Competing financial interests: The authors declare no competing financial interests

Reprints and permission information is available online at http://npg.nature.com/ reprintsandpermissions/

How to cite this article: Pinxterhuis, E. B. et al. Fast, greener and scalable direct coupling of organolithium compounds with no additional solvents. Nat. Commun. 7:11698 doi: $10.1038 /$ ncomms11698 (2016)

This work is licensed under a Creative Commons Attribution 4.0 International License. The images or other third party material in this article are included in the article's Creative Commons license, unless indicated otherwise in the credit line; if the material is not included under the Creative Commons license users will need to obtain permission from the license holder to reproduce the material To view a copy of this license, visit http://creativecommons.org/licenses/by/4.0/ 